\title{
Contingent access to stimuli associated with cocaine self-administration is required for reinstatement of drug-seeking behavior
}

\author{
JEFFREY W. GRIMM \\ National Institute on Drug Abuse, Baltimore, Maryland \\ and \\ PAUL J. KRUZICH and RONALD E. SEE \\ Medical University of South Carolina, Charleston, South Carolina
}

\begin{abstract}
In this study, we examined the importance of contingent access to a cocaine-related stimulus in the production of cocaine seeking following extinction of lever responding for cocaine. Rats self-administered cocaine for 2 weeks in daily 3-h sessions under a fixed-ratio 1 schedule. A compound stimulus (tone + light) was presented with each infusion. Following seven daily 3-h extinction sessions, rats were reintroduced to the compound stimulus alone. This stimulus was presented in three ways: (1) contingent on lever pressing, (2) noncontingent, and (3) both contingent and noncontingent. Following 3 more extinction days, rats were again reintroduced to the compound stimulus, yet with contingent access to cocaine. Only the two groups with contingent presentation increased lever responding on the 1 st day, while all groups increased responding for cocaine on the 2 nd day. The need for contingent access to drug-associated stimuli in the absence of drug to produce drug-seeking behavior may be relevant for studies of neural substrates of relapse.
\end{abstract}

Environmental stimuli, when paired with drug taking, may subsequently function as stimuli capable of eliciting craving in cocaine addicts (Ehrman, Robbins, Childress, \& O'Brien, 1992). Stimulus-induced craving has been correlated with physiological arousal (Ehrman et al., 1992; Liu, Vaupel, Grant, \& London, 1998) and neural activity in limbic regions (Childress et al., 1999; Grant et al., 1996). Several laboratories attempting to model cocainepaired stimulus conditioning in rats have met with mixed success (Arroyo, Markou, Robbins, \& Everitt, 1998; Davis \& Smith, 1976; de Wit \& Stewart, 1981; Tran-Nguyen et al., 1998; Weissenborn, Yackey, Koob, \& Weiss, 1995; Whitelaw, Markou, Robbins, \& Everitt, 1996).

We have previously utilized a method for modeling relapse in which rats press levers for cocaine injections and concurrently are presented with a compound stimulus composed of a visual and an auditory stimulus (Meil \& See, 1996). Following 14 self-administration days, rats experienced 21 extinction days during which lever pressing resulted in no consequences. This procedure was designed to model extended drug experience followed by protracted withdrawal. After extinction, subjects were allowed access to the compound stimulus, but not to cocaine. Rats pressed the previously cocaine-paired lever at levels significantly higher than the previous extinction session. The enhanced lever pressing after extinction is replic-

This study was supported by NIH Grant DA 10462 to R.E.S. Correspondence should be addressed to J. W. Grimm, Intramural Research Program, National Institute on Drug Abuse, P. O. Box 5180, Baltimore, MD 21224 (e-mail: jgrimm@intra.nida.nih.gov). able (Kruzich, Grimm, Rustay, Parks, \& See, 1999; See, Grimm, Kruzich, \& Rustay, 1999) and has been shown to involve the basolateral amygdala (Meil \& See, 1997).

One neglected aspect of procedures modeling relapse has been the importance of the contingency between a leverpress and stimulus presentation. In our initial studies (Meil \& See, 1996, 1997), animals showed elevated responding in the presence of both noncontingent and contingent presentations of a compound stimulus previously paired with drug infusions, complicating interpretation of the conditioned aspects of the stimulus. It was not clear whether rats were pressing due to the noncontingent presence of the stimulus (a conditioned response), or due to contingent presentations (presentation of a putative conditioned reinforcer). Distinguishing the nature of the response is critical, since interpretation of findings using such a paradigm in conjunction with neurobiological manipulations (e.g., lesions) will need to be made in the context of a conditioned response versus responding for a conditioned reinforcer. It is possible that such behaviors are mediated by separate neural substrates (McDonald \& White, 1993). In the present experiment, contingent and/ or noncontingent presentations of a compound stimulus were compared in order to explicitly assess the importance of contingent access to the cocaine-paired stimulus in relapse to drug-seeking behavior.

\section{METHOD}

\author{
Surgery \\ Male Sprague-Dawley rats (3-4 months; $350-450 \mathrm{~g}$ ) were singly \\ housed on a 12 -h reverse day/night cycle. All protocols were ap-
}


proved by an institutional animal care and use committee and followed the NIH Guide for the Care and Use of Laboratory Animals. Rats were anesthetized i.p. with ketamine $(100 \mathrm{mg} / \mathrm{kg})$, xylazine $(2 \mathrm{mg} / \mathrm{kg})$, and Equithesin $(0.5 \mathrm{ml} / \mathrm{kg})$. Silastic catheters were inserted into the right jugular vein and secured in place with silk suture. The tubing was subcutaneously run to an exit point on the back, where it was affixed to a 22-gauge threaded cannula for attachment to either an infusion line or an obturator. The catheter assembly was affixed to polypropylene mesh with cranioplastic cement. Rats were infused i.v. twice daily for 5 days with $6.67-\mathrm{mg}$ Timentin dissolved in $0.1-\mathrm{ml} 70-\mathrm{U} / \mathrm{ml}$ heparinized saline, followed by $0.1 \mathrm{ml}$ of $70-\mathrm{U} / \mathrm{ml}$ heparinized saline. During the remainder of the study, this infusion regimen occurred once following each self-administration session.

\section{Experimental Procedures}

Self-administration chambers contained two operant levers, white stimulus lights above each lever, and a red houselight on the opposite wall. The infusion line was attached to a liquid swivel and cocaine was delivered by an infusion pump. Rats were food deprived for $72 \mathrm{~h}$ and initially trained to press the right lever for food pellets. We have found that food restriction and lever training for food pellets facilitate acquisition of cocaine self-administration. Stimuli to be trained in conjunction with cocaine are not presented during food pellet training. Rats were then implanted with catheters and allowed 4 subsequent days of recovery prior to beginning cocaine selfadministration. Rats were maintained for the duration of the experiment at their weight immediately prior to surgery (approximately $85 \%-90 \%$ of free feeding) by restricting access to food in their home cages (we have recently found that rats will reinstate lever pressing in the presence of cocaine-paired stimuli without food deprivation or previous food pellet training [unpublished observations]). Rats self-administered in once daily 3 -h sessions. A right leverpress resulted in a compound stimulus presentation of the right stimulus light and a tone ( $2 \mathrm{kHz}, 15 \mathrm{~dB}$ above ambient). The compound (5-sec duration) occurred simultaneously with a cocaine injection $(0.33 \mathrm{mg} / 0.05 \mathrm{ml})$. Following stimuli presentation, a $35-\mathrm{sec}$ unsignaled time-out occurred. After 14 days of self-administration, animals were exposed to 7 days of daily 3 -h extinction sessions, where responding had no consequences. The subsequent day (Test Day 1), access to the tone + light was allowed, followed by 3 more days of extinction sessions. This initial day served as a test of the ability of the cocaine-paired stimuli to maintain leverpressing (drug seeking). The final day of the experiment (Test Day 2) was identical to Test Day 1, except that cocaine was delivered upon lever pressing. We used this test day as an index of the ability of cocaine itself to reinstate drug seeking (and further drug taking) following extinction training. Test days were $3 \mathrm{~h}$ in duration. Presentation of the cocaine-paired tone + light on Test Days 1 and 2 was varied in three groups as follows: (1) presentation contingent upon a right leverpress (contingent group), (2) noncontingent presentations every 10 min over the course of the session (noncontingent group), and (3) combined contingent and noncontingent presentations (combined group). The time-out period in the contingent groups was equivalent $(40 \mathrm{sec}$ total) to that found during the initial cocaine selfadministration sessions.

\section{Data Analysis}

The number of responses on the cocaine-paired right lever served as the primary dependent measure. All responses were recorded. Because the focus of this study was on responding for the cocainepaired stimuli on Test Day 1, responding on self-administration Extinction Days 7 and on Test Day 1 was examined both between and within all three groups using two-way repeated measures analysis of variance (two-way RM ANOVA). Cocaine consumption $(\mathrm{mg} / \mathrm{kg}$ ) on Test Day 2 (reintroduction of access to cocaine) and on Day 14 of self-administration (as a baseline) was compared between and within groups with a two-way RM ANOVA. The number of stimulus presentations on Test Day 1 was compared between groups with a one-way ANOVA. Post hoc comparisons were made with the Student-Newman-Keuls test and the criterion for statistical significance was $p<.05$.

\section{RESULTS}

Figure 1 illustrates right (active) lever responding over the course of the study. During the first few days of cocaine self-administration, several animals showed variable response patterns. By the end of the 1 st week, stable response rates were seen in all groups. Variability on the 1st day of extinction was high, yet there were no significant differences distinguishing the experimental groups [one-way ANOVA, $F(2,16)=2.26, p>.05$ ]. The mean number of daily responses on the nonreinforced lever was consistently low and did not differ significantly between groups or across the study (data not shown). Analysis of Extinction Day 7 and Test Day 1 responding with two-way RM ANOVA revealed a significant effect of treatment $[F(2,16)=8.22, p<.01]$, time $[F(1,15)=$ $18.40, p<.001]$, and a significant interaction $[F(2,15)=$ $8.42, p<.01]$. Results of post hoc analyses are presented in Figure 1. Only the combined and contingent groups significantly increased lever pressing on Test Day 1 relative to their respective Extinction Day 7 levels of responding. The combined and contingent groups each pressed significantly more than the noncontingent group on Test Day 1 . In addition, the contingent group responded significantly more than the combined group on Test Day 1. The number of stimulus presentations of Test Day 1 significantly differed between the combined group and the contingent and noncontingent groups $[F(2,16)=19.34$, $p<.001$; means \pm SEMs: combined, $28.43 \pm 2.34$; contingent, $16.33 \pm 1.23$; noncontingent, $16 \pm 0$ ]. The results of the two-way RM ANOVA for cocaine consumption on the last day of self-administration and on Test Day 2 revealed only a significant effect of time $[F(1,15)=21.26$, $p<.001]$. For all groups, more cocaine was consumed on Day 14 of self-administration versus Test Day 2 (overall means $\pm S E M \mathrm{~s}$ in $\mathrm{mg} / \mathrm{kg}$ : Day $14,33.74 \pm 2.58$; Test Day 2, 24.61 \pm 1.35 ).

\section{DISCUSSION}

As previously reported (Kruzich et al., 1999; Meil \& See, 1996, 1997; See et al., 1999), lever responding significantly increased during compound stimulus presentation after prolonged extinction. Contingent access to the stimulus resulted in robust increases in responding, while noncontingent stimulus presentations alone resulted in no such facilitation. This occurred with both contingent and noncontingent groups having received a comparable number of stimulus presentations. Moreover, contingent-only access resulted in higher responding than did combined contingent and noncontingent access. A reinstatement of cocaine consumption by all three groups indicates that the rats in the noncontingent group 


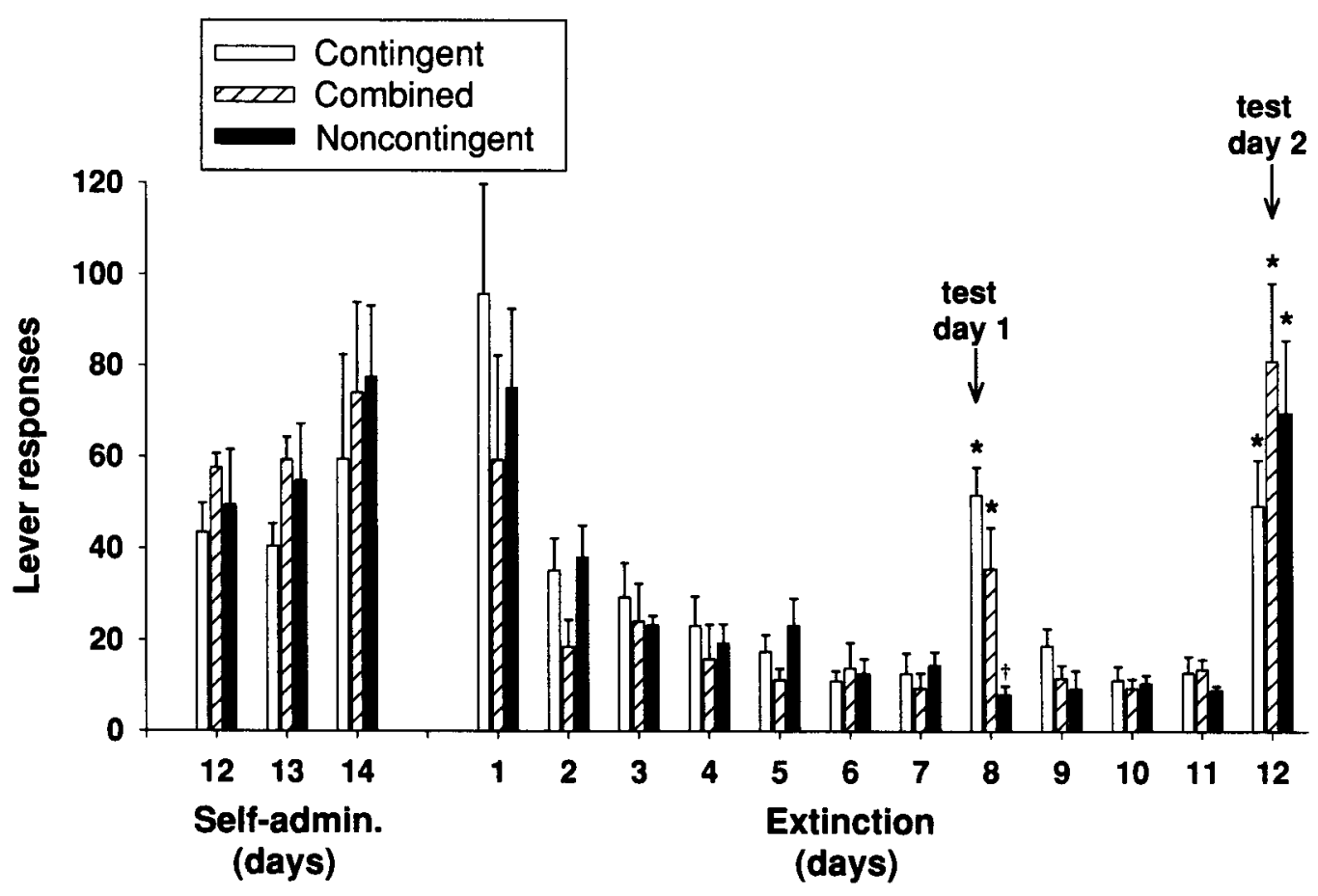

Figure 1. Right lever responses (mean $\pm S E M$ ) from rats trained with a compound stimulus and tested in conditions of contingent, noncontingent, or combined access to the compound stimulus ( $n=6-7$ per group). Test Day $1=$ tone + light alone. Test Day $2=$ tone + light and contingent cocaine. *Significant difference from the previous extinction day, $p<.05$. +Significant difference from the other two groups, $p<.05$.

failed to respond on Test Day 1 due to the lack of presentation of a contingent drug-paired stimulus, rather than due to an inability to respond. Overall, these results indicate that immediate stimulus presentation following a leverpress was necessary to drive robust reinstatement of responding following extinction.

Interpretation of responding in the presence of a stimulus previously paired with cocaine has often centered on a classical conditioned phenomenon. As yet, there are no animal models of relapse following chronic cocaine selfadministration that show consistent conditioned responding in the noncontingent presence of a conditioned stimulus. For example, noncontingent presentation of a light or a tone + light previously presented contingently with cocaine self-administration produced only slight or no elevations in responding when included as a component in extinction sessions (Tran-Nguyen et al., 1998; Weissenborn et al., 1995). In contrast, rats trained to respond for amphetamine or morphine responded at high levels for contingent presentations of a drug-paired tone following extinction (Davis \& Smith, 1976). These results do not necessarily indicate that noncontingent drug-paired stimulus presentations are without incentive motivational effects, but rather that the reinstatement model incorporating response-contingent presentations appears to be more sensitive to detecting these effects.

The importance of contingencies made apparent with the present data relates to concepts presented by Bindra
(1972) regarding an association model of operant conditioning. In particular, motivational changes are argued to be due to the learning of environmental contingencies. In the present context, a leverpress + compound stimulus presentation may serve as a "complete" conditioned stimulus (CS), with the actual proprioceptive feedback of the leverpress being a component of this stimulus critical for conditioning. A recent study by Johnson, Chen, Schmitz, Bordnick, and Shater (1998) provides an intriguing extension of this proposed relationship. They reported that the largest changes in physiological measures of arousal in cocaine-dependent subjects occurred in those individuals allowed to physically interact with cocaine-related paraphernalia, as opposed to audio or visual presentations alone. The drug-seeking behavior exhibited by rats may therefore best approximate craving due to the physical interaction with drug-related stimuli.

In any case, it appears that the lever responding in rats is increased by the compound stimulus presentations, due to a previous association with cocaine. Therefore, the behavior observed in this model is best described as responding for a conditioned reinforcer. Future studies employing this technique to investigate neurobiological substrates of relapse will need to interpret findings accordingly. In addition, the use of other procedures may prove useful for the development of relapse models, since a recent report showed the ability of a discriminative stimulus to elicit alcohol-seeking behavior in rats 
following prolonged extinction (Katner, Magalong, \& Weiss, 1999).

\section{REFERENCES}

Arroyo, M., Markou, A., Robbins, T. W., \& Everitt, B. J. (1998). Acquisition, maintenance and reinstatement of intravenous cocaine self-administration under a second-order schedule of reinforcement in rats: Effects of conditioned cues and continuous access to cocaine. Psychopharmacology, 140, 331-344.

BINDRA, D. (1972). A unified account of classical conditioning and operant training. In H. H. Black \& W. F. Prokasy (Eds.), Classical conditioning II: Current research and theory (pp. 453-481). New York: Appleton-Century-Crofts.

Childress, A. R., Mozley, P. D., McElgin, W., Fitzgerald, J., REIVICH, M., \& O'Brien, C. P. (1999). Limbic activation during cueinduced cocaine craving. American Journal of Psychiatry, 156, $11-18$

Davis, W. M., \& SMITH, S. G. (1976). Role of conditioned reinforcers in the initiation, maintenance and extinction of drug-seeking behavior. Pavlovian Journal of Biological Science, 11, 222-236.

DE Wir, H., \& STEWART, J. (1981). Reinstatement of cocaine-reinforced responding in the rat. Psychopharmacology, 75, 134-143.

Ehrman, R. N., Robbins, S. J., Childress, A. R., \& O'Brien, C. P. (1992). Conditioned responses to cocaine-related stimuli in cocaine abuse patients. Psychopharmacology, 107, 523-529.

Grant, S., London, E. D., Newlin, D. B., Villemagne, V. L., LiU, X., Contoreggi, C., Phillips, R. L., Kimes, A. S., \& Margolin, A. (1996). Activation of memory circuits during cue-elicited cocaine craving. Proceedings of the National Academy of Sciences, 93, 12040-12045.

Johnson, B. A., Chen, Y. R., SChmitz, J., Bordnick, P., \& Shater, A. (1998). Cue reactivity in cocaine-dependent subjects: Effects of cue type and cue modality. Addictive Behaviors, 23, 7-15.

Katner, S. N., Magalong, J. G., \& Weiss, F. (1999). Reinstatement of alcohol-seeking behavior by drug-associated discriminative stimuli after prolonged extinction in the rat. Neuropsychopharmacology, 20, 471-479.

Kruzich, P. J., Grimm, J. W., Rustay, N. R., Parks, C. D., \& See, R. E.
(1999). Predicting relapse to cocaine-seeking behavior: A multiple regression approach. Behavioural Pharmacology, 10, 513-521.

LiU, X., VAuPEL, D. B., GRant, S., \& London, E. D. (1998). Effect of cocaine-related environmental stimuli on the spontaneous electroencephalogram in polydrug abusers. Neuropsychopharmacology, 19 , 10-17.

McDonald, R. J., \& White, N. M. (1993). A triple dissociation of memory systems: Hippocampus, amygdala, and dorsal striatum. Behavioral Neuroscience, 107, 3-22.

MeIL, W. M., \& SEE, R. E. (1996). Conditioned cued recovery of responding following prolonged withdrawal from self-administered cocaine in rats: An animal model of relapse. Behavioural Pharmacology, 7, 754-763.

MeIL, W. M., \& SeE, R. E. (1997). Lesions of the basolateral amygdala abolish the ability of drug associated cues to reinstate responding during withdrawal from self-administered cocaine. Behavioural Brain Research, 87, 139-148.

See, R. E., Grimm, J. W., Kruzich, P. J., \& Rustay, N. (1999). The importance of a compound stimulus in conditioned drug-seeking behavior following one week of extinction from self-administered cocaine in rats. Drug \& Alcohol Dependence, 57, $41-49$.

Tran-Nguyen, T. L., Fuchs, R. A., Coffey, G. P., Baker, D. A., O'Del.l, L. E., \& Neisewander, J. L. (1998). Time-dependent changes in cocaine-seeking behavior and extracellular dopamine levels in the amygdala during cocaine withdrawal. Neuropsychopharmacology, 19, 48-59.

Weissenborn, R., YACKeY, M., KoOB, G. F., \& Werss, F. (1995). Measures of cocaine-seeking behavior using a multiple schedule of food and drug self-administration in rats. Drug \& Alcohol Dependence, 38, 237-246.

Whitelaw, R. B., Markou, A., Robbins, T. W., \& Everitt, B. J. (1996). Excitotoxic lesions of the basolateral amygdala impair the acquisition of cocaine-seeking behaviour under a second-order schedule of reinforcement. Psychopharmacology, 127, 213-224.

(Manuscript received November 17, 1999; revision accepted for publication April 4, 2000.) 